\title{
Postoperative EGFR-TKI treatment on residual multiple ground-glass nodules after resection for lung adenocarcinoma: a rare case report and literature review
} \author{
6007-1247

\section{Xuefei Shi} \\ Huzhou Central Hospital

\section{Weiwei Min} \\ Huzhou Central Hospital

\section{Caihua Yu} \\ Huzhou Central Hospital \\ Hongwei Li \\ Huzhou Central Hospital
}

Jianbin Zhang ( $\nabla$ fishing11@163.com )

HuZhou Central Hospital, Affiliated Central Hospital of Huzhou University https://orcid.org/0000-0001-

\section{Case report}

Keywords: multiple ground-glass nodules (mGGNs), synchronous multiple primary lung cancer (SMPLC), EGFR-TKI

Posted Date: March 26th, 2021

DOI: https://doi.org/10.21203/rs.3.rs-317254/v1

License: (a) (1) This work is licensed under a Creative Commons Attribution 4.0 International License. Read Full License 


\section{Abstract}

Background: Multiple ground-glass nodules (mGGNs) in the lung has been defined as synchronous multiple primary lung cancer (SMPLC), but it is very difficult to differentiat SMPLC from intrapulmonary metastases, and the treatment is still controversial. Target therapy on residual mGGNs after resection for the dominant lesion might be a selectable approach.

Case presentation: We report a rare case of lung adenocarcinoma displaying primary EGFR-T790M mutation, in which the patient underwent radical resection of lesions in the left upper lung(LUL), and continued osimertinib treatment for the residual mGGNs in all lobes of the right lung. The mGGNs in the right lung displayed different responses to osimertinib.

Conclusions: This case indicated that intropulmonary spread might be coexisted with multicentric origin for mGGNs.

\section{Background}

Although mGGNs in the lung has been defined as synchronous multiple primary lung cancer (SMPLC) [1] [2], it is very difficult to differentiat SMPLC from intrapulmonary metastases, and the treatment is still controversial[3]. Genetic analyse has been proved useful in the discrimination [4][5], but it is impractical for each tumor. we presented a novel strategy in the treatment of bilateral mGGNs displaying different responses to Osimertinib.

\section{Case Presentation}

A 50-year-old woman was admitted to our hospital because of bilateral mGGNs detected on routine health checkup. She had no history of smoking, no history of malignant tumors, and no family history of lung cancer. The chest computed tomography (CT) revealed a 1.6-cm-diameter irregularly shaped lesion in the LUL, and $28 \mathrm{GGNs}$ in all lobes of the right lung(Fig. 1). Brain MRI and abdominal ultrasound detected no distant metastases. The possibility of SMPLC was considered, but it was very difficult to differentiate it from a primary lung cancer with intrapulmonary metastases. The patient refused to perform lung puncture biopsy to identify the pathology. In March 2019, thoracoscopic left $S^{(1+2+3)}$ segmentectomy and systematic lymph node dissection were performed, the patient made an uneventful recovery. Postoperative pathology revealed pulmonary adenocarcinomas in the LUL, all resected lymph nodes were negative (Fig. 2). Aggressive surgical treatment or stereotactic body radiation therapy (SBRT) was not feasible for this case, and the patient refused to undergo chemotherapy. So the EGFR testing was performed, and it manifested as a primary T790M mutation. After the multiple disciplinary team (MDT), target therapy was adopted on the consent of the patient. In March 2019, the patient was treated with 80 mg Osimertinib daily. After 3 months of therapy, the chest CT showed that most GGNs in the right lung were partial absorbed. Thus the final diagnosis might be a primary lung cancer with intropulmonary spread coexisting with multicentric origin. Osimertinib treatment was continued. Until the last follow-up in 
October 2020, the reviewed chest CT showed that most GGNs in the right lung were completely absorbed, only one GGN was radiologically same as before (Fig. 3). The patient's condition remained stable, with no signs of recurrence and metastases, she is currently receiving Osimertinib treatment.

\section{Discussion And Conclusions}

With the improvement of public health awareness and the popularization of thin-section CT, the detective rate of SMPLC with mGGNs is increasing[6][7]. Distinguishing SMPLC from intrapulmonary metastases is very difficult without surgical procedure, but it is critical because the therapeutic approach for these two conditions is entirely different[8][9]. In our case, the preoperative chest CT showed mGGNs in both lungs, with the dominant lesion in the LUL. The SMPLC was firstly considered, and the indication for surgery was clear. Postoperative pathology confirmed lung adenocarcinoma for the dominant lesion. The patient refused to undergo partial resection of the right lung for clarification. The most likely diagnosis was SMPLC, but it cannot completely rule out intrapulmonary metastasis.

Currently there is no uniform standard of surgical indication and procedure for SMPLC with mGGNs[11]. One or two-stage surgical resection has been the most effective treatment[12][13]. SBRT can be considered to achieve local control [14] [15]. Few cases of chemotherapy or target therapy have been reported, Ye etal.[16] reported a successful case of treatment for multiple pulmonary nodules. They performed surgical resection for the gefitinib insensitive lesion, and continued target therapy for sensitive lesions. Cheng etal.[17] reported a successful experience of target therapy on residual mGGNs after resection for lung adenocarcinoma. In our case, aggressive treatment was not feasible because of 28 lesions in all lobes of the right lung. There was no method to distinguish a primary lung cancer from intrapulmonary metastasis. Oral Osimertinib was adopted as target therapy. To our surprise, lesions in the right lung displayed different responses to Osimertinib. To the best of our knowledge, this is the first report of an integrated approach using surgical treatment combined with target therapy for mGGNs. It indicated that intropulmonary spread might be coexisted with multicentric origin for mGGNs. In conclusion, we report a successful strategy on the postoperative treatment for mGGNs.

\section{Declarations}

\section{Ethics approval and consent to participate}

The article was reviewed and approved by the by the research ethics committee of Huzhou Central Hospital, Affiliated Central Hospital of HuZhou University. Written informed consent was signed by the participant.

\section{Consent for publication}

Not applicable.

\section{Availability of data and materials}


All data generated or analysed during this study are included in this article.

\section{Competing interests}

The authors declare that they have no competing interests.

\section{Funding}

Not applicable.

\section{Authors' contributions}

Jianbin Zhang performed the operation ang postoperative treatment on this patient, and was the major contributor in writing. Xuefei Shi provided the photos editing and language polishing. Caihua Yu and Weiwei Min were involved in patient's care and follow-up. Hongwei Li performed the technical guidance. All authors read and approved final manuscripts.

\section{Acknowledgements}

Not applicable.

\section{Abbreviations}

mGGNs: multiple ground-glass nodules; SMPLC: synchronous multiple primary lung cancer; LUL: left upper lung; CT: computed tomography; SBRT: stereotactic body radiation therapy; MDT: multiple disciplinary team.

\section{References}

1. Naidich DP, Bankier AA, MacMahon $\mathrm{H}$, et al. Recommendations for the management of subsolid pulmonary nodules detected at CT: a statement from the Fleischner Society. Radiology. 2013;266:304-17.

2. AJCC Cancer. Staging Manual. 8th ed. New York: Springer; 2017.

3. Dai C, Ren Y, Xie H, et al. Clinical and radiological features of synchronous pure ground-glass nodules observed along with operable non-small cell lung cancer. J Surg Oncol. 2016;113:738-44.

4. Liu M, He WX, Song N, et al. Discrepancy of epidermal growth factor receptor mutation in lung adenocarcinoma presenting as multiple ground-glass opacities. Eur $\mathrm{J}$ Cardiothorac Surg. 2016;50:909-13.

5. Zhou X, Tian L, Fan J, et al. Method for discriminating synchronous multiple lung cancers of the same histological type: miRNA expression analysis. Medicine (Baltimore). 2016;95:e4478.

6. Vazquez M, Carter D, Brambilla E, et al. Solitary and multiple resected adenocarcinomas after CT screening for lung cancer: histopathologic features and their prognostic implications. Lung Cancer. 
2009;64:148-54.

7. Tanvetyanon T, Boyle TA. Clinical implications of genetic heterogeneity in multifocal pulmonary adenocarcinomas. J Thorac Dis. 2016;8:E1734-8.

8. Arai J, Tsuchiya T, Oikawa M, et al. Clinical and molecular analysis of synchronous double lung cancers. Lung Cancer. 2012;77:281-7.

9. Zhang Z, Gao S, Mao Y, et al. Surgical Outcomes of Synchronous Multiple Primary Non-Small Cell Lung Cancers. Sci Rep. 2016;6:23252.

10. Chung J-H, Choe G, Jheon S, et al. Epidermal Growth Factor Receptor Mutation and PathologicRadiologic Correlation Between Multiple Lung Nodules with Ground-Glass Opacity Differentiates Multicentric Origin from Intrapulmonary Spread. J Thorac Oncol. 2009;4:1490-5.

11. Sihoe AD, Cardillo G. Solitary pulmonary ground-glass opacity: is it time for new surgical guidelines? Eur J Cardiothorac Surg. 2017;52:848-51.

12. Sihoe AD, Van Schil P. Non-small cell lung cancer: when to offer sublobar resection. Lung Cancer. 2014;86:115.

13. Shi Z, Chen C, Jiang S, et al. Uniportal video-assisted thoracic surgery resection of small groundglass opacities (GGOs) localized with CT-guided placement of microcoils and palpation. J Thorac Dis. 2016;8:1837-40.

14. Creach KM, Bradley JD, Mahasittiwat $P$, et al. Stereotactic body radiation therapy in the treatment of multiple primary lung cancers. Radiother Oncol. 2012;104:19-22.

15. Donovan EK, Swaminath A. Stereotactic body radiation therapy (SBRT) in the management of nonsmall-cell lung cancer: Clinical impact and patient perspectives. Lung Cancer. 2018;9:13-23.

16. Chenyang Ye J, Wang MD, Wenshan Li, et al. Novel Strategy for Synchronous Multiple Primary Lung Cancer Displaying Unique Molecular Profiles. Ann Thorac Surg. 2016;101:e45-7.

17. Cheng B, Li C, Zhao Y, et al. The impact of postoperative EGFR-TKIs treatment on residual GGO lesions after resection for lung cancer. Signal Transduction Targeted Therapy. 2021;6:73.

\section{Figures}
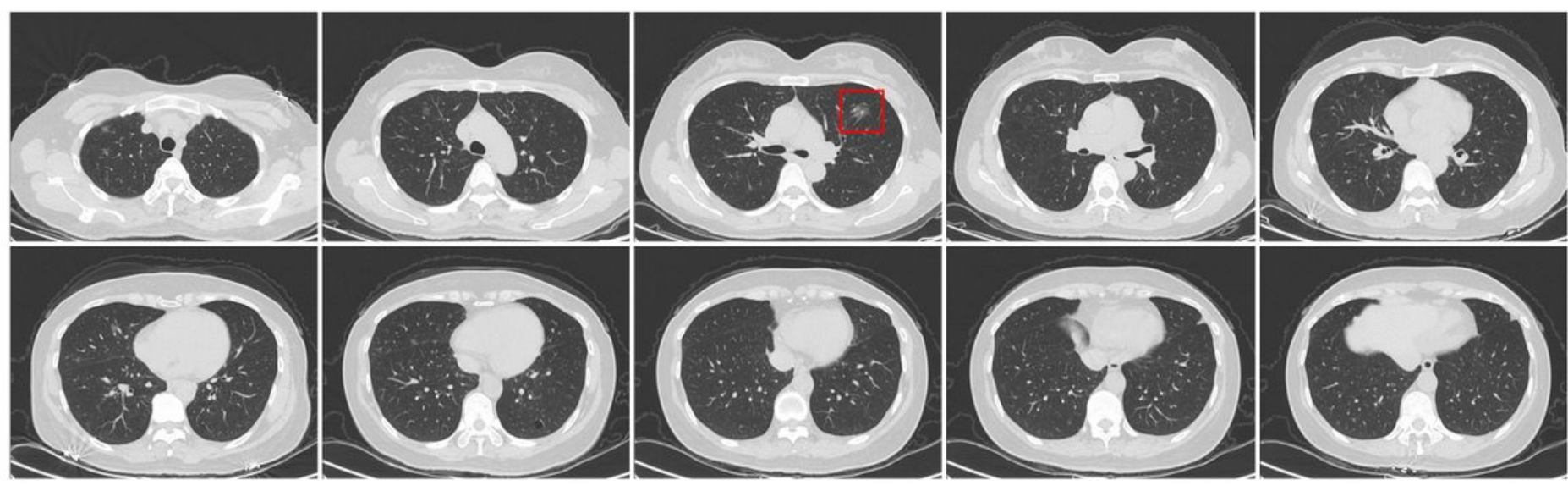
Figure 1

The preoperative computed tomography scanning. A $1.6-\mathrm{cm}$ diameter irregularly shaped lesion in the LUL, and $\mathrm{mGGNs}$ in all lobes of the right lung.

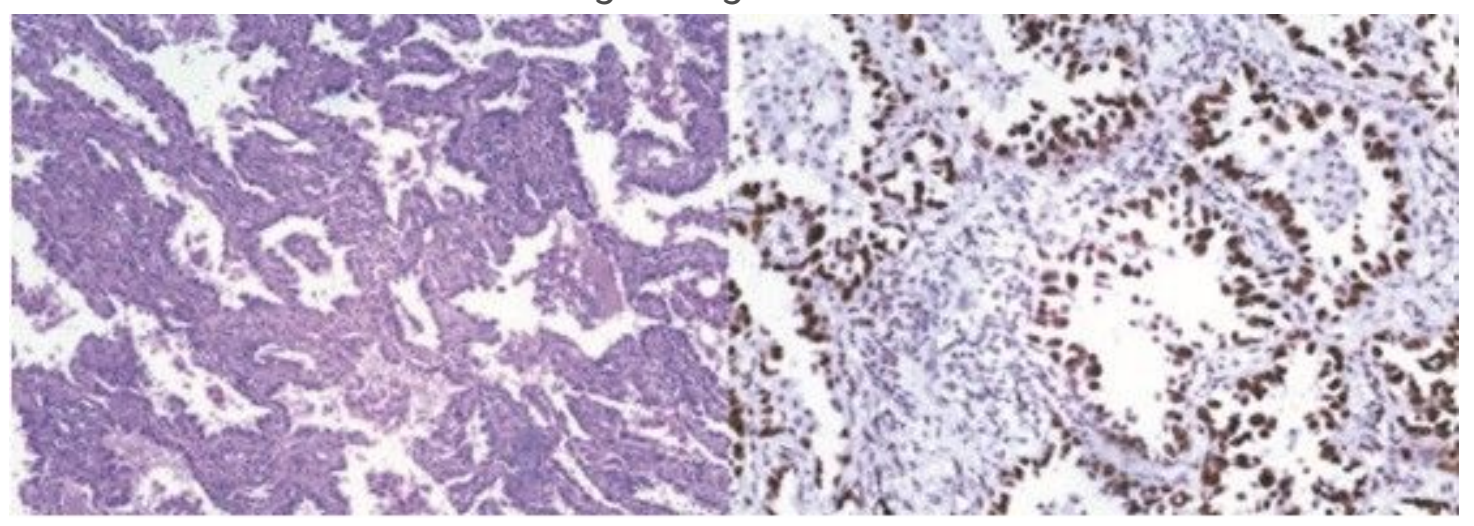

Figure 2

The postoperative pathology. Acinar pulmonary adenocarcinoma with negative resected lymph nodes.
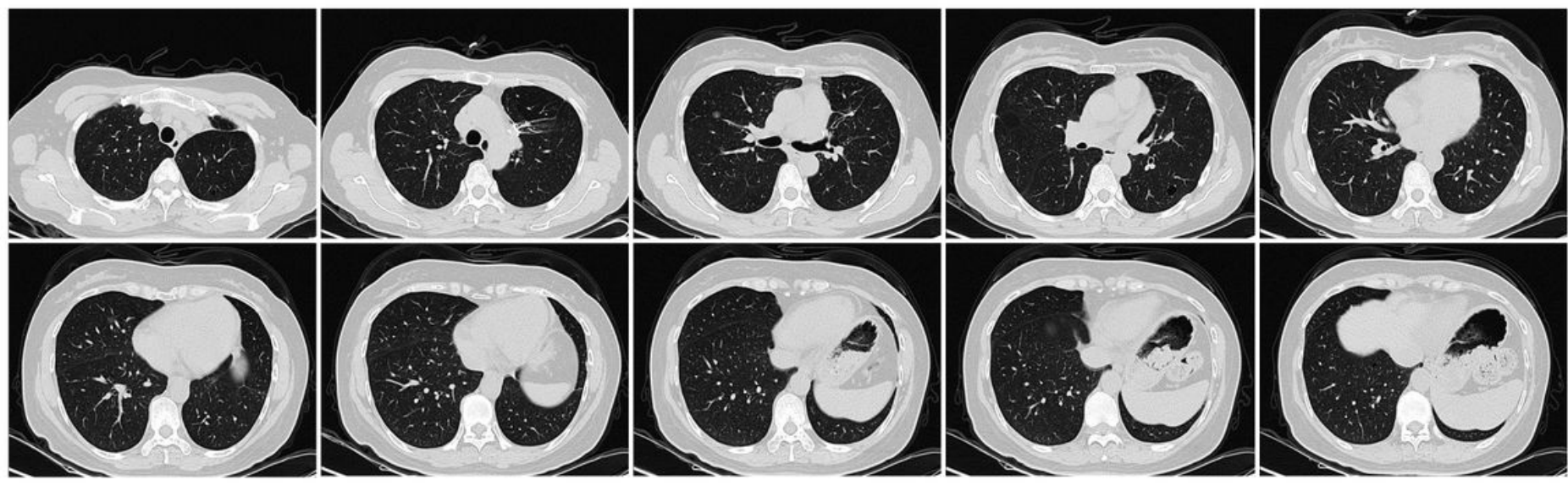

Figure 3

The postoperative computed tomography scanning. After nineteen months of target therapy, most mGGNs in the right lung were completely absorbed, only one GGN in the right upper lung was radiologically same as before.

\section{Supplementary Files}

This is a list of supplementary files associated with this preprint. Click to download.

- CAREchecklistEnglish2013.pdf 